\title{
Calcium Carbonate Scale Formation, Prediction and Treatment (Case Study Gumry Oilfield-PDOC)
}

\author{
A. Saifelnasr ${ }^{1}$, M. Bakheit ${ }^{1}$, K. Kamal ${ }^{2, *}$, A. Lila ${ }^{3}$ \\ ${ }^{1}$ College of Science, Neelain University, Khartoum, Sudan \\ ${ }^{2}$ College of Science, Bahri University, Khartoum, Sudan \\ ${ }^{3}$ Central Petroleum Laboratory, Sudan \\ *E-mail address: kamaltha99@rediffmail.com
}

\begin{abstract}
In this study the predictions and probabilities of calcium carbonate scale formation and corrosion occurrence inside Gumry Field Process Facilities (GFPF) and produced water injection process units (PWTP) was carried out. This was done calculating the Langelier Saturation Index (LSI), Ryznar Stability Index (RSI), Puckorius Scaling Index (PSI). The samples results for LSI index and PSI index were compared and found that all samples under threaten of calcium carbonate scale. The samples results for RSI index and found that all samples under formation of heavy calcium carbonate scale. Special excel computer program was designed to calculate the (LSI, RSI, PSI). The scale was cleaned and removed by $10 \%$ diluted hydrochloric acid and commercial scale inhibitor.
\end{abstract}

Keywords: scale formation; LSI; RSI; PSI indices

\section{INTRODUCTION}

The production of water is natural consequence of the production of oil and gas from subterranean reservoirs. Water that is present in these reservoirs contains dissolved salts and dissolved gases. Under certain conditions these salts precipitate and become Scale deposits [1]. It is not possible to produce oil and gas for any significant time before water is also produced .The water /oil boundary will rise over time and channeling from water permeable zones will cause increased water to be produced. Scales are formed due to precipitation and crystal growth at the water surface. Precipitation is due to low solubility or excess amounts of salts. The scale forming salt in heat exchangers and boilers are those having retrograde solubility with temperature [2].

The scale formation and corrosivity is qualitatively predicted using various indices $[3,4]$ that depend upon water parameters. The Langelier Saturation Index (LSI) [5] is an indicator of cooling water scale potential. This index indicates the driving force for scale formation and growth in terms of $\mathrm{pH}$ as a master variable. It is calculated the value of alkalinity $\left(\mathrm{mg} \mathrm{L}^{-1}\right.$ as $\mathrm{CaCO}_{3}$ or calcite), the calcium hardness $\left(\mathrm{mg} \mathrm{L}^{-1} \mathrm{Ca}^{2+}\right.$ as $\left.\mathrm{CaCO}_{3}\right)$, the total dissolved solids ( $\left.\mathrm{mg} \mathrm{L}^{-1} \mathrm{TDS}\right)$, the actual $\mathrm{pH}$, and the temperature of the water $\left({ }^{\circ} \mathrm{C}\right)$. 


$$
\mathrm{LSI}=\mathrm{pH}-\mathrm{pH}_{\mathrm{s}}
$$

where $\mathrm{pH}$ is the actual $\mathrm{pH}$ and $\mathrm{pH}_{\mathrm{s}}$ is the saturation $\mathrm{pH}$.

The $\mathrm{pH}_{\mathrm{s}}$ can be calculated from the relation, $\mathrm{pH}_{\mathrm{s}}=(9.30+\mathrm{A}+\mathrm{B})-(\mathrm{C}+\mathrm{D})$, where

$$
\begin{aligned}
& A=\frac{(\log [T D S]-1)}{10}, \\
& B=-13.12 x \log \left({ }^{\circ} \mathrm{C}+273\right)+34.55, \\
& C=\log \left[\mathrm{Ca}^{+2} \text { asCaCO}_{3}\right]-0.4, \\
& D=\log [\text { alkaalinityas CaCO }]
\end{aligned}
$$

Thus a negative LSI indicates no potential to scale, the water will dissolve $\mathrm{CaCO}_{3}$, while a positive value indicates $\mathrm{CaCO}_{3}$ precipitation. Ryznar Stability Index (RSI) [6] is given by: $\mathrm{RSI}=2 \mathrm{pH}_{\mathrm{s}}-\mathrm{pH}$, whereas $\mathrm{RSI}>6$ indicates corrosion while $\mathrm{RSI}<6$ indicates scale formation. Puckorius Scaling Index (PSI) [7] is obtained from the relation: $\mathrm{PSI}=2 \mathrm{pH}_{\mathrm{s}}-\mathrm{pH}_{\mathrm{e}}$, whre $\mathrm{pH}_{\mathrm{eq}}=1.465 \times \log _{10}$ [Alkalinity $]+4.54$ and $[$ Alkalinity $]=\left[\mathrm{HCO}_{3}{ }^{-}\right]+2\left[\mathrm{CO}_{3}{ }^{2-}\right]+$ $\left[\mathrm{OH}^{-}\right]$. It has the same indication as RSI.

Scale and corrosion continue to be serious and costly problems in gas and oil wells and production facilities. Scale commonly occurs in multiple locations within a production system. These locations include production and injection wells, flow lines, valves, vessels, separators, heat exchangers and pumps.

The result of scale deposition in oil and gas production systems is increased costs owing to equipment failures, manpower-related maintenance of scaled equipment, and lost production. Scale formation increases when active sites are there due to the a direct crystal growth without crystal formation. "Scale inhibitors don't prevent precipitation, they delay the inevitable by extending induction time." [8,9]

$$
\text { Equation Time }=\frac{[\text { inhibitor }] \mathrm{M}}{\mathrm{k}[\mathrm{SR}-1] \mathrm{P}-1}
$$

where Time is the induction time

inhibitor is the scale inhibitor molar concentration

$\mathbf{M}$ is s coefficient

$\mathbf{k}$ is a temperature dependent rate constant

SR is the saturation ratio

$\mathbf{P}$ is the number of molecules in a critical sized cluster

Dosage models have been used successfully to prevent scale in cooling systems, reverse osmosis, oil field and mining applications.

The impact of common scale inhibitors on induction time can be modeled by adding an inhibitor term to a classic model for induction time [10,11] 
It must be noted that there is a maximum saturation level beyond which inhibitors will not prevent scale by this mechanism at any dosage. This is typically $150 \mathrm{x}$ saturation for calcite.

The calcium carbonate depositions in Produced Water Package Treatment (PWPT) used for injection purpose and $\mathrm{pH}$, temperature, total suspending solids (TSS), Total Dissolve Solids (TDS),), Alkalinity, Calcium Hardness, conductivity were determined and the indices, LSI, RSI and PSI were calculated using excel program.

\section{MATERIALS \& METHODS}

Analar grade chemicals were used as received. Twenty four untreated raw produced water was collected from the sample point at Gumry FPF, Sudan, on 25th -June-2009 and same in 25th of January 2010 at 9:00AM.

Temperature was determined using probe (model testo 915-1, $\mathrm{pH}$ with $\mathrm{pH}$ meter (Wagtech UK), TDS, turbidity, conductivity were determined using Hanna instruments. Total hardness and calcium were determined by EDTA titration. Alkalinity was obtained by titration against standard sulfuric acid.

\section{RESULTS \& DISCUSSION}

The Physicochemical properties for Gumry produced water is given in Table A.

Table A. Gumry produced water package treatment PWT outlet results.

\begin{tabular}{|c|c|c|c|}
\hline \multicolumn{2}{|c|}{ Test } & Result & Unit \\
\hline \multicolumn{2}{|c|}{$\mathrm{pH}$} & 6.37 & \\
\hline \multicolumn{2}{|c|}{ Turbidity } & 11.17 & NTU \\
\hline \multicolumn{2}{|c|}{ Total Suspending Solids } & 4.00 & ppm \\
\hline \multicolumn{2}{|c|}{ Total Dissolved Salts } & 3330 & ppm \\
\hline \multicolumn{2}{|c|}{ Alkalinity } & 4630.05 & ppm \\
\hline \multicolumn{2}{|c|}{ Conductivity } & 6320 & $\mu \mathrm{s} / \mathrm{cm}$ \\
\hline \multirow{2}{*}{ Hardness } & total & 57.80 & ppm \\
\hline & temporary & 48.60 & ppm \\
\hline
\end{tabular}

The indices LSI, RSI and PSI are calculated using the data of Table 1. According to the results of (OGMs) in Table 1 for samples collected in the year 2009 and Table 2 for samples 
collected in the year 2010, (Langelier Saturation Index) LSI prediction that all samples under slight calcium carbonate $\left(\mathrm{CaCO}_{3}\right)$ scale forming conditions. In the Table 1 Oil Gathering Manifold 2 (OGM-2), shows negative LSI reading (-0.04) which indicate of (slightly corrosive but non scale forming), changed to $(+0.34)$ in Table 2 year 2010 which means (slightly scale forming and corrosive).

Interpretation of the Langelier Saturation Index (Carrier, 1965) [12]

\begin{tabular}{|c|c|c|c|c|c|}
\hline LSI Index & 2.0 & 0.5 & 0.02 & -0.5 & -2.0 \\
\hline $\begin{array}{c}\text { Value } \\
\text { Indication }\end{array}$ & $\begin{array}{c}\text { Scale forming but } \\
\text { non corrosive }\end{array}$ & $\begin{array}{c}\text { Slightly scale } \\
\text { forming and } \\
\text { corrosive }\end{array}$ & $\begin{array}{c}\text { Balanced but } \\
\text { pitting } \\
\text { corrosion } \\
\text { possible }\end{array}$ & $\begin{array}{c}\text { Slightly } \\
\text { corrosive but } \\
\text { non-scale } \\
\text { forming }\end{array}$ & $\begin{array}{c}\text { Serious } \\
\text { corrosion }\end{array}$ \\
\hline
\end{tabular}

The Ryznar Stability index (RSI) predict that all samples collected in the s 2009 and 2010 were under threat of (heavy calcium carbonate $\left(\mathrm{CaCO}_{3}\right)$ scale forming). All the samples RSI changed from the range (5to 6) which means and predict (light scale) in the year 2009 to the range RSI (4 to 5) in the year 2010 which indicates and predicts (heavy scale) and expect more complicated problem of scale buildup in the coming years if no real treatments or chemical additives are used.

Risk of emergency shutdown for long time will reduce pressure, release soluble acid gases, and increase $\mathrm{pH}$; all these factors activate calcium carbonate scale to form and the system will face serious problems in the next staring.

Interpretation of the Ryznar Stability Index (Carrier, 1965)

\begin{tabular}{|c|c|c|c|c|c|c|}
\hline RSI Value & $4.0-5.0$ & $5.0-6.0$ & $6.0-7.0$ & $7.0-7.5$ & $7.5-9.0$ & $>9.0$ \\
\hline Indication & $\begin{array}{c}\text { Heavy } \\
\text { scale }\end{array}$ & Light scale & $\begin{array}{c}\text { little scale } \\
\text { or } \\
\text { corrosion }\end{array}$ & $\begin{array}{c}\text { Corrosion } \\
\text { significant }\end{array}$ & $\begin{array}{c}\text { Heavy } \\
\text { corrosion }\end{array}$ & $\begin{array}{c}\text { Corrosion } \\
\text { intolerable }\end{array}$ \\
\hline
\end{tabular}

The PSI values also predicts that all samples witch collected in the year 2009 and 2010 were under heavy calcium carbonate $\left(\mathrm{CaCO}_{3}\right)$ scale formation.

So that all prediction factors (LSI, RSI, PSI) clear that the Gumry plant, oil productive Wells, produced water treatment system, Crude-Crude heaters, Export Tanks and heat Exchangers under threaten of heavy calcium carbonate scale forming.

The presence of high concentration of carbon dioxide $\left(\mathrm{CO}_{2}\right)$ will sustainable the system without Scale for long time due to reduction in water $\mathrm{PH}$ and also the presence of high pressure in the system. 
Table 1. Gumry Oil Gathering Manifolds (OGM) (2009).

\begin{tabular}{|c|c|c|c|c|c|c|c|c|c|c|c|}
\hline 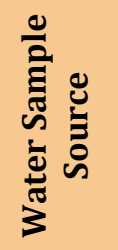 & 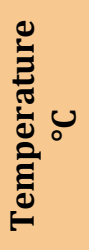 & 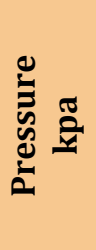 & 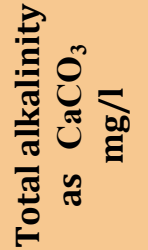 & 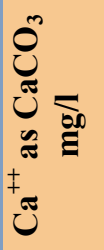 & 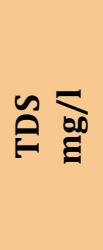 & 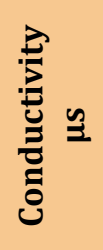 & $\frac{n}{2}$ & $\pi$ & $\overline{3}$ & $\overline{\bar{n}}$ & $\bar{n}$ \\
\hline OGM-2 & 66.7 & 686 & 2500 & 30 & 2590 & 5240 & 6.41 & 6.35 & -0.04 & 6.46 & 3.3 \\
\hline OGM-3 & 59.1 & 687 & 2700 & 40 & 2570 & 5120 & 6.209 & 6.32 & $\begin{array}{c}+0.1 \\
1\end{array}$ & 6.09 & 2.9 \\
\hline OGM-4 & 81.6 & 689 & 5000 & 46 & 2725 & 8890 & 6.67 & 6.8 & $\begin{array}{c}+1.1 \\
2\end{array}$ & 4.55 & 1.4 \\
\hline OGM-5 & 82.1 & 685 & 5100 & 45 & 4200 & 8500 & 5.69 & 6.46 & $\begin{array}{c}+0.7 \\
6\end{array}$ & 4.93 & 1.4 \\
\hline $\begin{array}{c}\text { FPF } \\
\text { INLET }\end{array}$ & 74 & 638 & 4800 & 45 & 3930 & 7840 & 5.8 & 6.5 & $\begin{array}{c}+0.6 \\
5\end{array}$ & 5.19 & 1.76 \\
\hline
\end{tabular}

Table 2. Gumry Oil Gathering Manifolds (OGM) (2010).

\begin{tabular}{|c|c|c|c|c|c|c|c|c|c|c|c|}
\hline 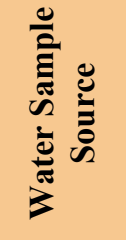 & 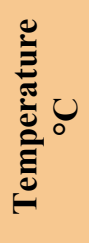 & 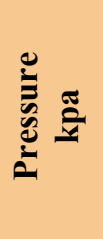 & 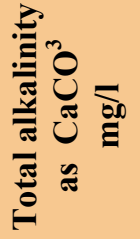 & 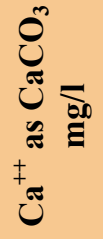 & 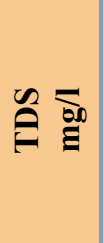 & 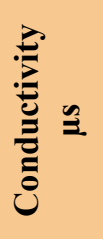 & $\stackrel{n}{a}$ & $\bar{a}$ & $\bar{n}$ & $\bar{n}$ & $\overline{\mathscr{2}}$ \\
\hline OGM-2 & 68.1 & 672 & 3000 & 66 & 3640 & 7280 & 6.00 & 6.32 & +0.34 & 5.63 & 2.23 \\
\hline OGM-3 & 67.8 & 675 & 2500 & 66.6 & 3420 & 6809 & 6.05 & 6.23 & +0.17 & 5.88 & 2.66 \\
\hline OGM-4 & 83.9 & 672 & 3250 & 66.6 & 5740 & 11470 & 5.7 & 6.75 & +1.04 & 4.65 & 1.72 \\
\hline OGM-5 & 80.5 & 671 & 4500 & 65.5 & 5650 & 11310 & 5.6 & 6.45 & +0.88 & 4.79 & 1.35 \\
\hline $\begin{array}{c}\text { FPF } \\
\text { INLET }\end{array}$ & 75 & 640 & 5750 & 74.5 & 5580 & 11170 & 5.55 & 6.5 & +0.95 & 4.59 & 1.05 \\
\hline
\end{tabular}

In Table 3 and Table 4 first and second stage separators the factors (LSI, RSI and PSI) predict the risk of scale which may buildup in the water outlet inside Separators will cause 
reduction in the pipe diameter, that cause to the bad water separations and prolong separation time.

Thermodynamic considerations (pressure drop) activate scale forming and buildup, temperature increased in the second stage separators, heat exchangers so that increasing temperature will increase scale tendency in the mentioned facilities and will be under threaten of scale forming.

Table 3. Gumry FPF first and second stage Separators Samples (2009).

\begin{tabular}{|c|c|c|c|c|c|c|c|c|c|c|c|}
\hline 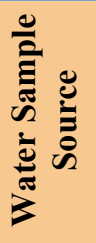 & 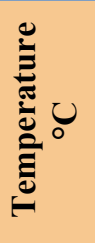 & 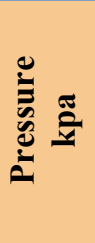 & 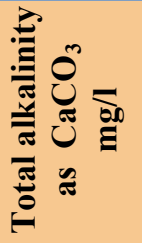 & 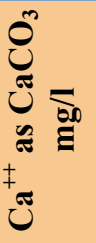 & 饮 & 疍 & $\stackrel{a}{a}$ & $\underline{T}$ & $\bar{\Omega}$ & $\overline{\mathscr{z}}$ & $\overline{\mathscr{Q}}$ \\
\hline $1^{\mathrm{ST}} \mathrm{A}$ & 74 & 535 & 4700 & 50 & 3825 & 7650 & 5.8 & 6.51 & +0.69 & 5.12 & 1.7 \\
\hline $1^{\mathrm{ST}} B$ & 74 & 533 & 4800 & 60 & 3740 & 7490 & 5.7 & 6.4 & +0.68 & 5.04 & 1.51 \\
\hline $1^{\mathrm{ST}} \mathrm{C}$ & 74 & 533 & 4700 & 100 & 3750 & 7500 & 5.5 & 6.5 & +0.99 & 4.5 & 1.09 \\
\hline $1^{\mathrm{ST}} \mathrm{D}$ & 74 & 534 & 4700 & 55 & 3730 & 7530 & 5.75 & 6.5 & +0.7 & 5.03 & 1.61 \\
\hline $2^{\mathrm{ND}} \mathrm{A}$ & 81 & 270 & 4500 & 25 & 3800 & 7600 & 6.01 & 6.2 & +0.19 & 5.8 & 2.14 \\
\hline $2^{\mathrm{ND}} B$ & 82 & 274 & 4600 & 25 & 3650 & 7300 & 5.98 & 6.2 & +0.21 & 5.77 & 2.07 \\
\hline $2^{\mathrm{ND}} \mathrm{C}$ & 82 & 274 & 4800 & 30 & 3740 & 7720 & 5.88 & 6.15 & +0.26 & 5.62 & 1.85 \\
\hline $2^{\mathrm{ND}} \mathrm{D}$ & 81 & 280 & 4600 & 35 & 3680 & 7120 & 5.85 & 6.25 & +0.39 & 5.46 & 1.81 \\
\hline
\end{tabular}

FPF=Field Process Facilities

Table 4. Gumry FPF first and second stage Separators Samples (2010).

\begin{tabular}{|c|c|c|c|c|c|c|c|c|c|c|c|}
\hline 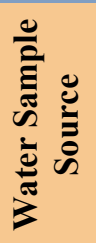 & 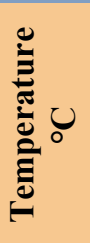 & 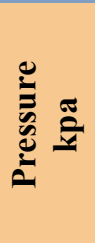 & 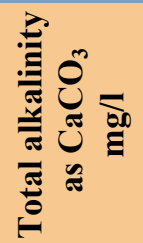 & 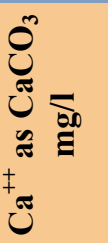 & $\stackrel{\mathscr{2}}{\hat{E}}$ & 忌 & $\stackrel{n}{a}$ & $\frac{\pi}{2}$ & $\overline{\boldsymbol{\Omega}}$ & $\tilde{\mathscr{A}}$ & $\overline{\mathscr{2}}$ \\
\hline $1^{\text {st }} \mathrm{A}$ & 75.1 & 535 & 5700 & 74 & 5580 & 11170 & 5.55 & 6.5 & +0.94 & 4.6 & 1.06 \\
\hline $1^{\mathrm{ST}} \mathrm{B}$ & 75.3 & 531 & 5450 & 74.5 & 5650 & 11240 & 5.566 & 6.36 & +0.79 & 4.77 & 1.12 \\
\hline $1^{\text {st }} \mathrm{C}$ & 75.1 & 531 & 5700 & 74.3 & 5560 & 11200 & 5.55 & 6.41 & +0.86 & 4.69 & 1.06 \\
\hline $1^{\text {st }} \mathrm{D}$ & 75.3 & 527 & 5700 & 74.5 & 5700 & 11630 & 5.54 & 6.46 & +0.91 & 4.63 & 1.05 \\
\hline $2^{\text {nd }} A$ & 81.5 & 291 & 5550 & 74.5 & 5570 & 11140 & 5.45 & 6.26 & +0.79 & 4.66 & 0.89 \\
\hline $2^{\text {nd }} B$ & 81.5 & 289 & 5500 & 74.5 & 5590 & 11170 & 5.46 & 6.19 & +0.72 & 4.73 & 0.90 \\
\hline $2^{\text {nd }} C$ & 82 & 290 & 5350 & 74.5 & 5580 & 11240 & 5.46 & 6.27 & +0.80 & 4.66 & 0.90 \\
\hline $2^{\text {nd }} D$ & 82.1 & 282 & 5650 & 74.5 & 5550 & 11180 & 5.43 & 6.36 & +0.92 & 4.51 & 0.84 \\
\hline
\end{tabular}


FPF=Field Process Facilities

In Table 5 and Table 6 predict of the risk of scale buildup inside Heat Exchangers (A, B, $\mathrm{C}, \mathrm{D})$, will effect in reducing heat transfer between hot oil and cold oil due to the increase in the pipe wall thickness which caused by calcium carbonate scale layer and that leading to increase energy consumptions and problems of back pressure.

Table 5. Gumry Heat Exchangers Samples (2010).

\begin{tabular}{|c|c|c|c|c|c|c|c|c|c|c|c|}
\hline 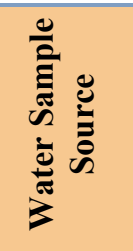 & 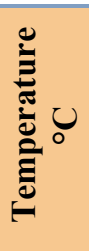 & 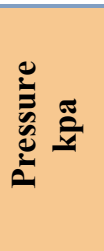 & 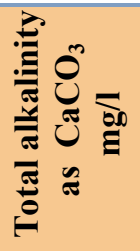 & 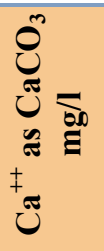 & 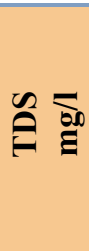 & 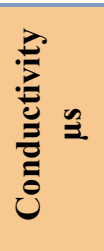 & $\stackrel{a}{a}$ & 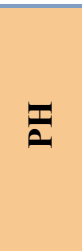 & $\bar{a}$ & $\overline{\mathscr{A}}$ & $\overline{\mathscr{\Omega}}$ \\
\hline H.EX.A & 78.2 & 355 & 5500 & 74.5 & 5570 & 11140 & 5.51 & 6.26 & +0.75 & 4.77 & 1.01 \\
\hline H.EX.B & 78 & 355 & 5500 & 74.5 & 5590 & 11170 & 5.51 & 6.19 & +0.67 & 4.84 & 1.02 \\
\hline H.EX.C & 78.2 & 355 & 5350 & 74.5 & 5580 & 11240 & 5.52 & 6.27 & +0.74 & 4.78 & 1.05 \\
\hline H,EX.D & 78.3 & 344 & 5650 & 74.5 & 5550 & 11180 & 5.50 & 6.36 & +0.86 & 4.64 & 0.97 \\
\hline
\end{tabular}

H.EX=Heat Exchanger (A, B, C, D)

Table 6. Gumry Heat Exchangers Samples (2009).

\begin{tabular}{|c|c|c|c|c|c|c|c|c|c|c|c|}
\hline 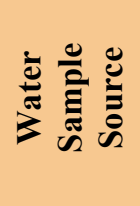 & 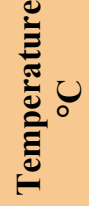 & 站 & 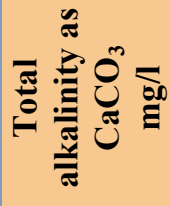 & 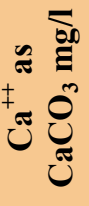 & 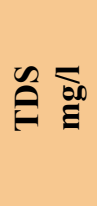 & 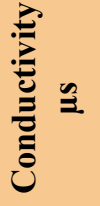 & $\frac{n}{2}$ & $\underline{a}$ & $\bar{\Omega}$ & $\tilde{\mathscr{Z}}$ & $\overline{\mathscr{2}}$ \\
\hline H.EX.A & 77.2 & 355 & 4500 & 25 & 3800 & 7600 & 6.07 & 6.2 & +0.12 & 5.95 & 2.26 \\
\hline H.EX.B & 77 & 355 & 4600 & 25 & 3650 & 7300 & 6.06 & 6.05 & +0.02 & 6.06 & 2.23 \\
\hline H.EX.C & 77.3 & 355 & 4800 & 30 & 3740 & 7720 & 5.96 & 6.25 & +0.28 & 5.65 & $\begin{array}{c}1.99 \\
9\end{array}$ \\
\hline H,EX.D & 77.1 & 344 & 4600 & 35 & 3680 & 7120 & 5.92 & 6.25 & +0.33 & 5.59 & 1.91 \\
\hline
\end{tabular}

H.EX=Heat Exchanger (A, B, C, D)

Table 7. Gumry Crude-Crude Heaters Samples (2009).

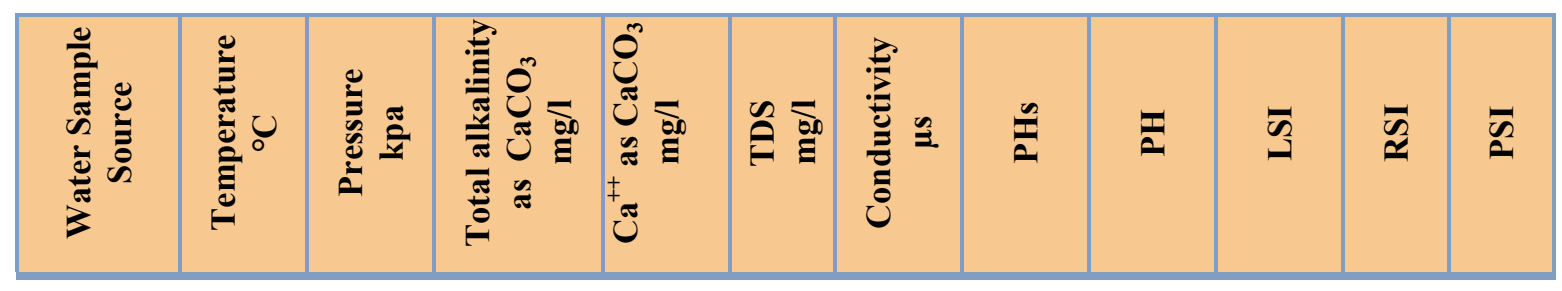




\begin{tabular}{|l|c|c|c|c|c|c|c|c|c|c|c|}
\hline C.H.A & 81.4 & 305 & 4500 & 25 & 3800 & 7600 & 6.00 & 6.5 & +0.49 & 5.51 & 2.1 \\
\hline C.H.B & 85 & 310 & 4600 & 25 & 3650 & 7300 & 5.93 & 6.4 & +0.46 & 5.47 & 1.97 \\
\hline C.H.C & 80.1 & 310 & 4800 & 30 & 3700 & 7720 & 5.92 & 6.4 & +0.47 & 5.44 & 1.90 \\
\hline C.H.D & 80 & 315 & 4600 & 35 & 3680 & 7120 & 5.87 & 6.5 & +0.63 & 5.24 & 1.84 \\
\hline
\end{tabular}

Table 8. Gumry Crude-Crude Heaters Samples (2010).

\begin{tabular}{|c|c|c|c|c|c|c|c|c|c|c|c|}
\hline 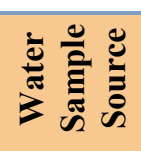 & 产晜。 & 产 & 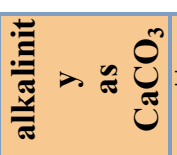 & 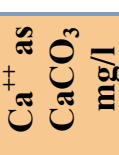 & Oิ & 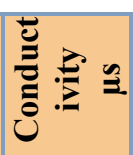 & $\stackrel{\mathscr{a}}{\mathbf{a}}$ & $\underline{a}$ & $\overline{3}$ & $\bar{\approx}$ & $\bar{n}$ \\
\hline C.H.A & 81.8 & 320 & 5700 & 74 & 5580 & 11170 & 5.44 & 6.5 & +1.06 & 4.38 & 0.85 \\
\hline С.Н.В & 82 & 327 & 5450 & 74.5 & 5650 & 11240 & 5.45 & 6.56 & +1.10 & 4.36 & 0.90 \\
\hline C.H.C & 82 & 327 & 5700 & 74.3 & 5560 & 11200 & 5.43 & 6.41 & +0.97 & 4.46 & 0.84 \\
\hline C.H.D & 81.6 & 327 & 5700 & 74.5 & 5700 & 11630 & 5.44 & 6.46 & +1.01 & 4.42 & 0.85 \\
\hline
\end{tabular}

Table 9. Gumry FPF Tanks Samples (2010).

\begin{tabular}{|c|c|c|c|c|c|c|c|c|c|c|c|}
\hline 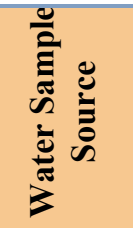 & 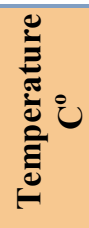 & 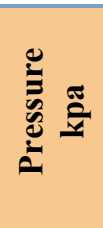 & 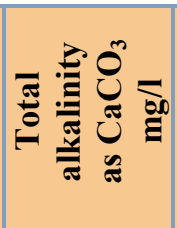 & 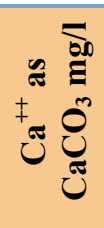 & 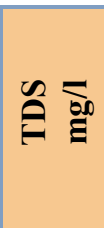 & 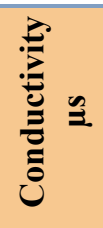 & $\stackrel{n}{a}$ & $\bar{a}$ & $\sqrt{3}$ & $\overline{\mathscr{n}}$ & $\overline{\mathscr{E}}$ \\
\hline EX.T.A & 73 & A.P & 5750 & 70 & 3300 & 6610 & 5.58 & 6.82 & +1.24 & 4.34 & 1.12 \\
\hline EX.T.B & 74 & A.P & 5750 & 70 & 3300 & 6600 & 5.87 & 6.83 & +1.26 & 4.31 & 1.09 \\
\hline PWTA & 77 & A.P & 4630 & 57 & 3330 & 6660 & 5.70 & 6.46 & +0.76 & 4.94 & 1.50 \\
\hline PWTB & 77 & A.P & 4630 & 57 & 3330 & 6660 & 5.7 & 6.46 & +0.76 & 4.90 & 1.50 \\
\hline
\end{tabular}

EX.T=Export Tank (A, B), PWT=Produced Water Tank (A, B).

Table 10. Gumry FPF Tanks Samples (2009).

\begin{tabular}{|c|c|c|c|c|c|c|c|c|c|c|c|}
\hline 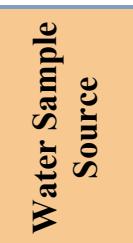 & 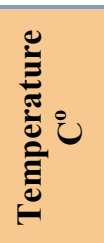 & 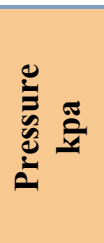 & 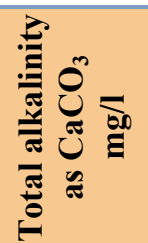 & 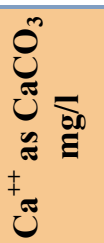 & 气ิ & 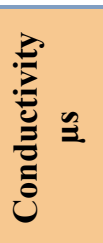 & 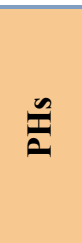 & $\frac{\pi}{2}$ & $\bar{\Omega}$ & $\overline{\mathscr{A}}$ & $\bar{n}$ \\
\hline EX.T.A & 74.5 & A.P & 4300 & 50 & 3740 & 7460 & 5.83 & 6.85 & +1.01 & 4.82 & 1.81 \\
\hline EX.T.B & 73.5 & A.P & 4500 & 70 & 3550 & 7002 & 5.68 & 6.83 & +1.14 & 4.54 & 1.48 \\
\hline
\end{tabular}




\begin{tabular}{|c|c|c|c|c|c|c|c|c|c|c|c|}
\hline PWTA & 73.3 & A.P & 4700 & 60 & 3850 & 7720 & 5.74 & 6.5 & +0.76 & 4.98 & 1.56 \\
\hline PWTB & 72 & A.P & 4600 & 55 & 3780 & 7520 & 5.80 & 6.5 & +0.69 & 5.11 & 1.71 \\
\hline
\end{tabular}

EX.T=Export Tank (A, B), PWT=Produced Water Tank (A, B).

In the Table 7 and 8 increase temperature activates the tendency to form calcium carbonate scale in the crude oil heaters due to the decrease of solubility with temperature increase. In the Table 9 and Table 10, pressure drops to atmospheric pressure inside the Tanks would permit to release dissolved acid gases $\mathrm{CO}_{2}$ from the fluid phases and that reduces the solubility of gases, increase $\mathrm{pH}$ of the produced water so all these parameters increase calcium carbonate $\mathrm{CaCO}_{3}$ Scale to form. LSI increased in the year 2010 is an indication of increasing tendency of calcium carbonate scale forming; see the variations in Figures 1 and 2, high values of LSI in the tanks with low pressure it is good situation for scale forming.

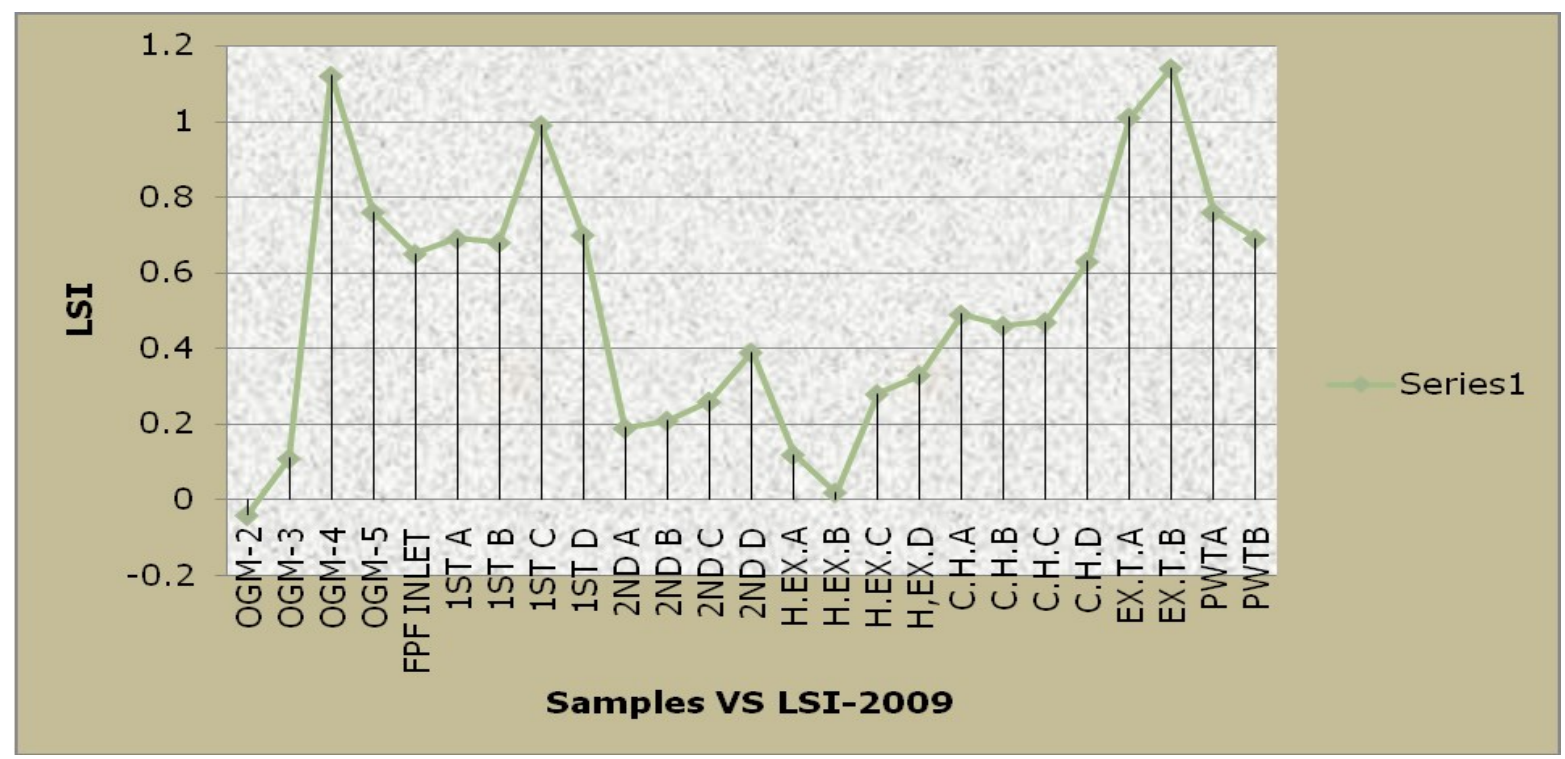

Fig. 1. The trend of LSI 2009. 


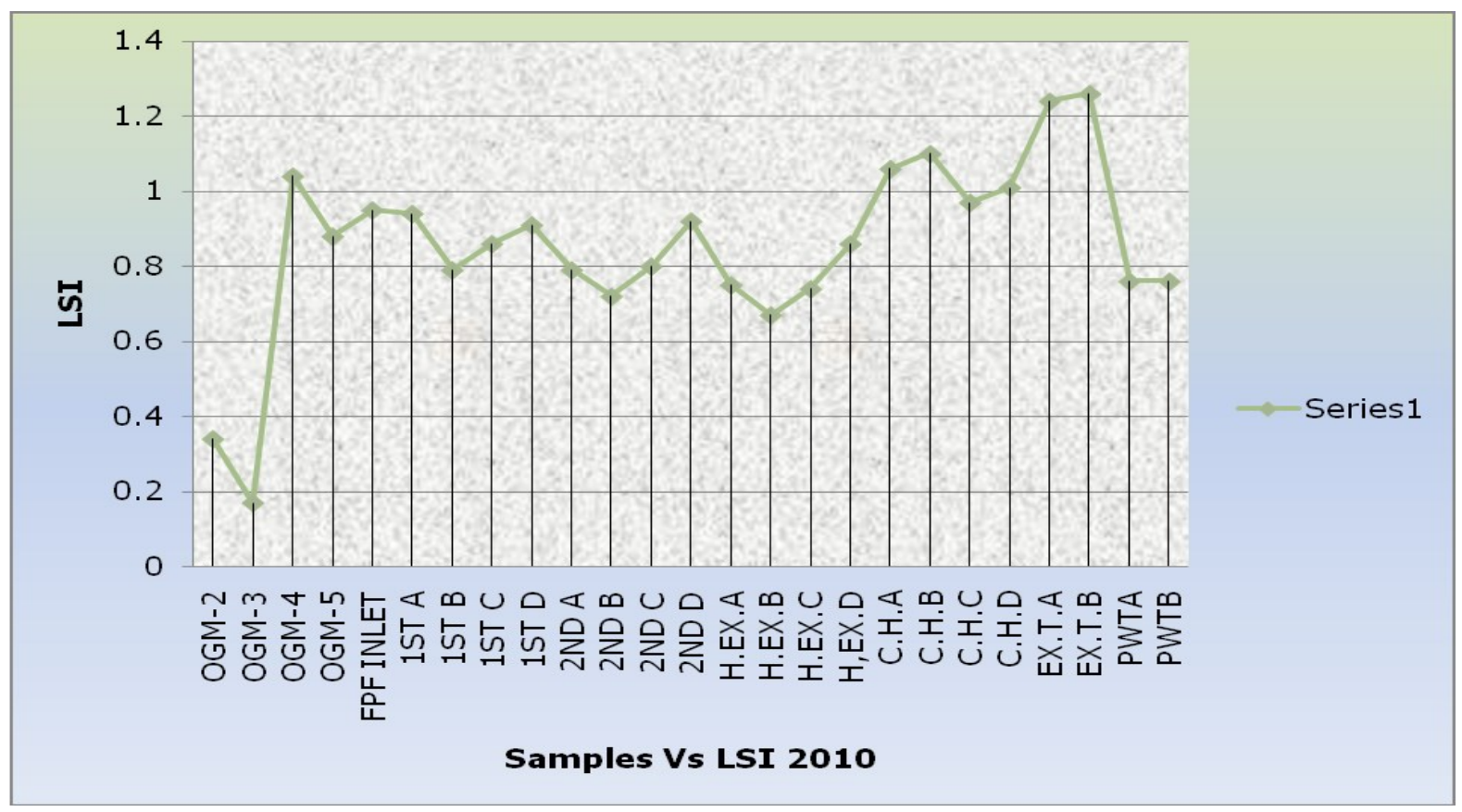

Fig. 2. The trend of LSI 2010.

In Fig. 3 and Fig. 4 the Ryznar Stability index (RSI) predict that all samples which collected in the year 2009 and which in the year 2010 were under threaten of ( heavy calcium carbonate $\left(\mathrm{CaCO}_{3}\right)$ scale forming). All the samples RSI changed from the range (5 to 6) which means and predict (light scale) in the year 2009 to the range RSI (4 to 5) in the year 2010 which means and predict (heavy scale) except OGM-2 and OGM-3 (2010), expect more complicated problem of scale buildup in the coming years 2011, 2012. If no real treatments or chemical additives done.

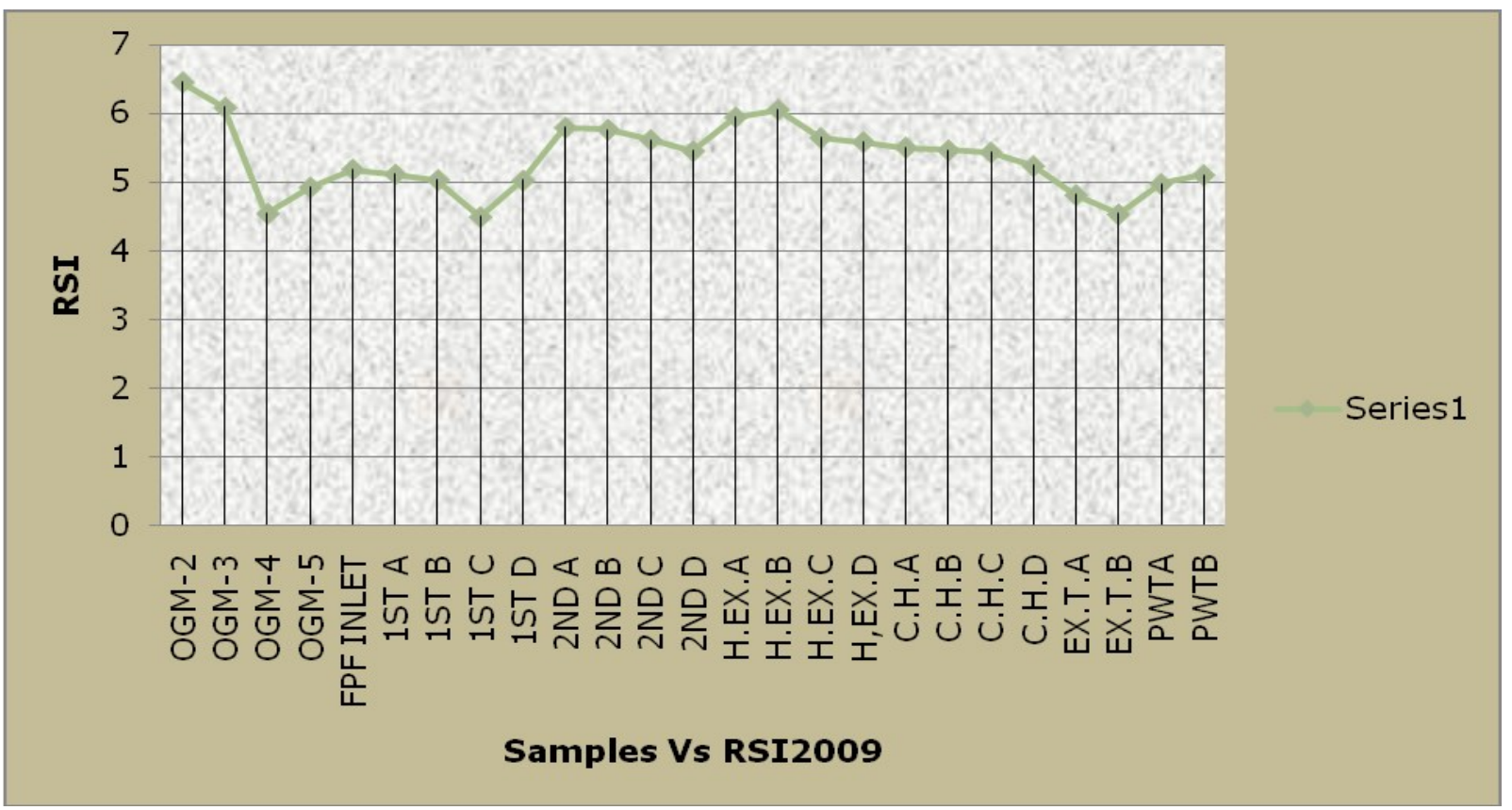

Fig. 3. The trend of RSI 2009. 


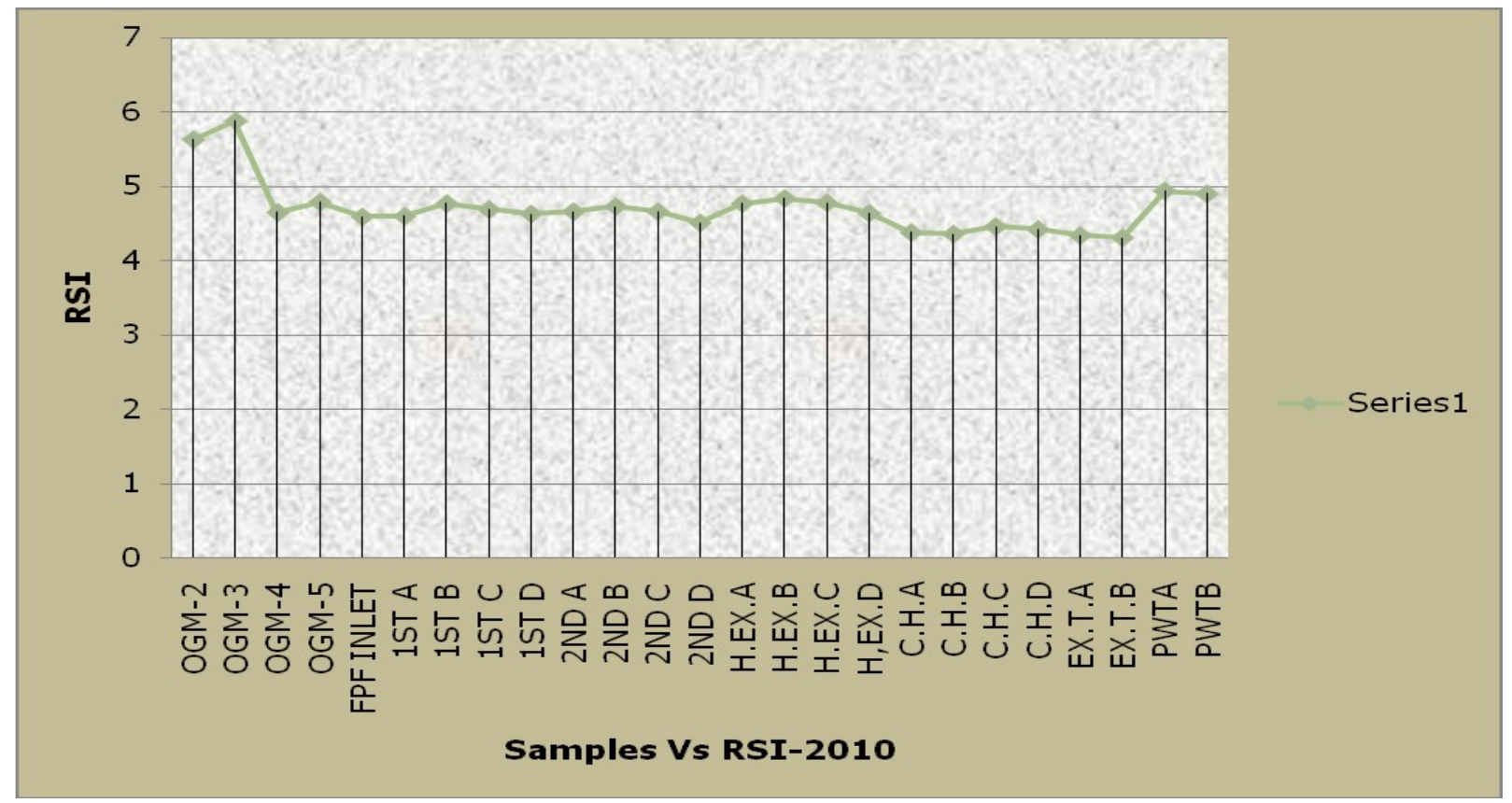

Fig. 4. The trend of RSI 2010.

In Figure 5 and Figure 6, PSI predicts that all samples witch collected in the year 2009 and 2010 were under heavy calcium carbonate $\left(\mathrm{CaCO}_{3}\right)$ scale forming, all PSI reading less than (6) which means that the water under tendency of forming calcium carbonate scale.

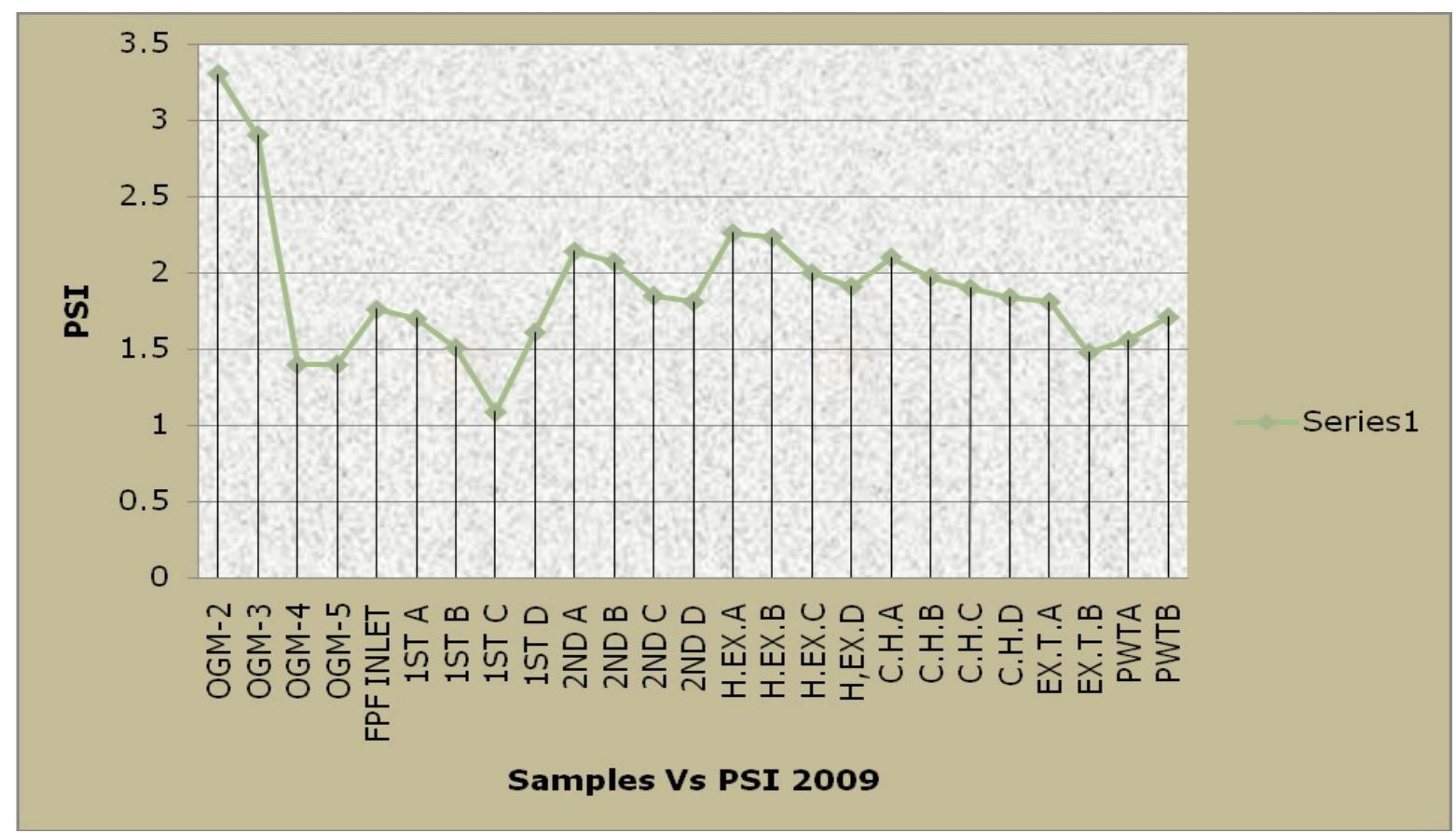

Fig. 5. The trend of PSI 2009. 


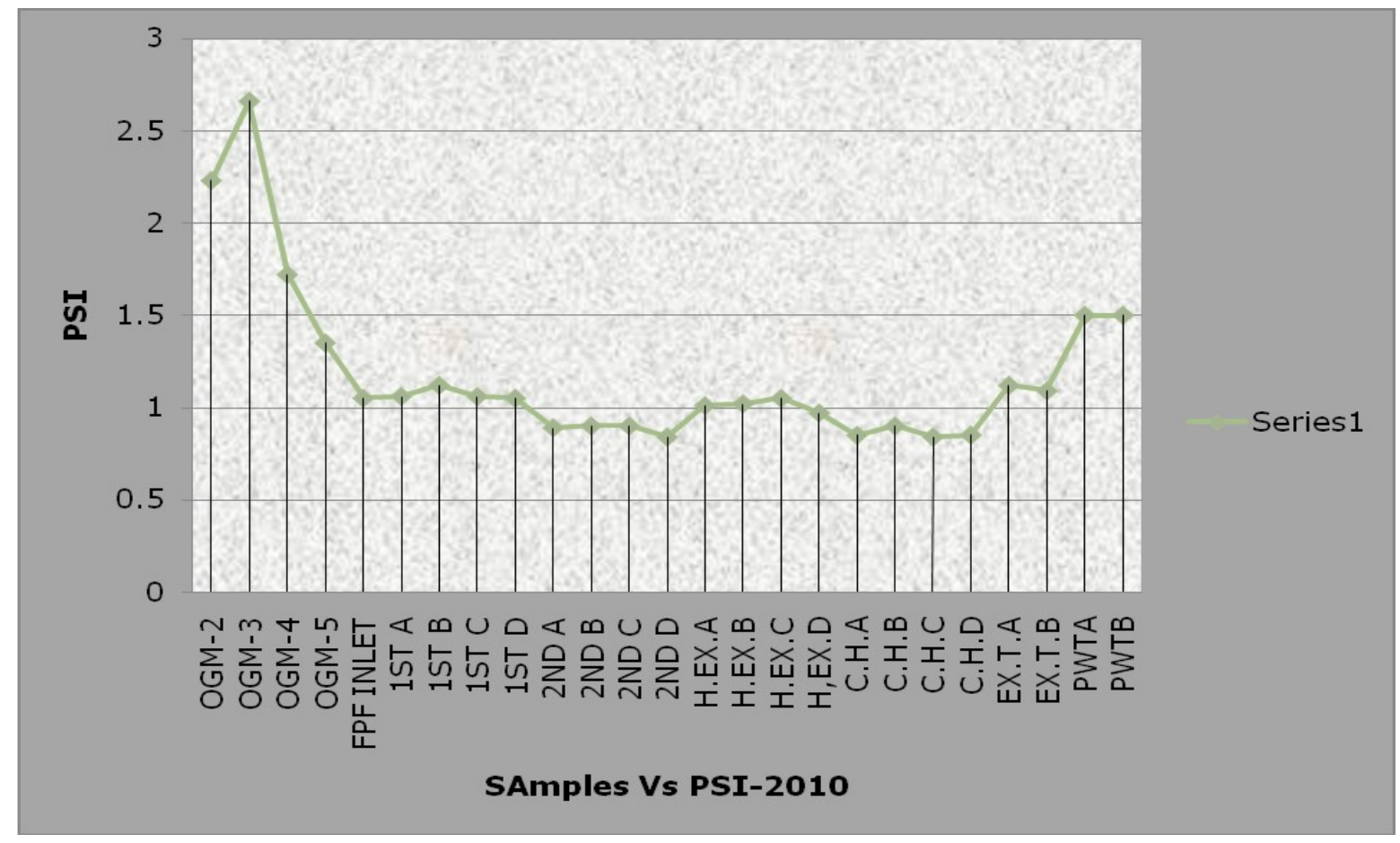

Fig. 6. The trend of PSI 2010.

\section{CONCLUSIONS}

1. Many locations in Gumry oil field under threatened by calcium carbonate scale in future:

2. Application the special Excel program to calculate Langelier Saturation Index (LSI), Ryznar Stability Index (RSI) and Puckorius Scaling Index (PSI) depend on (Carrier, 1965) equation and can be applied in water industries.

3. In order to obtain proper prevention treatment, recommended that to start scale inhibitor immediately inside Gumry FPF to protect from scale accumulation and buildup.

\section{References}

[1] Introduction to water treatment, Vol. II (American Water Work Association], 1984, 118

[2] Handbook of Industrial water Conditioning, 9th edition, Betz. Laboratories Inc., Trevose, USA), 1991, 118.

[3] Puchorius P. R., Brooke J. M. (1990). A new index for CaCO3 scale prediction in cooling water systems, (paper No. 90) presented at the NACE-corrosion Conf., Las Vegas, USA, 23-27 April 1990.

[4] Puchorius P. R., Brooke J. M. (1988). Effectively evaluating cooling water programme, (paper No. 17) presented at the NACE-corrosion Conf., St. Louis, USA, 21 - 25 March 1988.

[5] Langelier W. F., JAWWA 28(10) (1936) 1500-1521. 
[6] Ryznar J. W., JAWWA 36 (1944) 472.

[7] P. Puckorius, Get A Better Reading on Scaling Tendency of Cooling Water, Power, p. 79-81, September, 1983.

[8] Ferguson R. J. (1992), Developing Scale Inhibitor Models, WATERTECH, Houston, TX.

[9] Ferguson R. (2011), Thermodynamics and Kinetics of Cooling Water Treatment, Association of Water Technologies.

[10] Ferguson R. J., A Kinetic Model for Calcium Carbonate Scale, CORROSION/84, Paper No. 46, (Houston, TX:NACE INTERNATIONAL 1984).

[11] Ferguson R. J., Developing Scale Inhibitor Models, WATERTECH, Houston, TX, $1992.12,13$

[12] Carrier Air Conditioning Company, 1965. Handbook of Air Conditioning System Design. McGraw-Hill Books. New York. 